\title{
P63 \& KI67 ANTIGEN EXPRESSION IN RELATION TO THE AGGRESSIVENESS OF ORAL SQUAMOUS CELL CARCINOMA
}

\author{
Mohamed Abdelrahman Mohamed", Kamal Abdelrahman Kamal **, \\ Amr Saad Abdelwahab ${ }^{* *}$ and Magdi Alazazi ${ }^{* * *}$
}

\begin{abstract}
Aim: To investigate the expression of $\mathrm{p} 63$ protein and ki67 antigen in a series of graded OSCC to evaluate the potential association between the expression of the two markers and the aggressiveness of histologic grades of OSCC. Methods: Hematoxylin and Eosin (H\&E) and streptavidin-biotin immunoperoxidase staining techniques was used to detect the expression of p63 \& ki67 in different grades of OSCC. Evaluation of immunostaining was done using computer image analyzer system \& statistical analysis. Results: regarding p63 \& ki67 expression were obtained by comparing the area $\%$ between different grading of OSSC, there was a statistically highly significant difference between different grades of OSCC $(\mathrm{P}<0.01) \&$ there was a statistically significant direct correlation between p63 \& ki67 stain. Conclusion: These results suggest that p63 \& ki67 could reflect biological features, such as proliferation and differentiation for recognition of clinical behavior of OSCC also p63 \& ki67 alteration may be associated with the progression of these neoplasms.
\end{abstract}

Keywords: OSCC, immunohistochemical markers, p63, ki67.

\section{INTRODUCTION}

Oral carcinogenesis is a multi-step process involving gene mutations and chromosomal abnormalities ${ }^{(1)}$. The transition from normal oral epithelium to oral dysplasia and cancer results from accumulated genetic and epigenetic alterations ${ }^{(2)}$. Oral squamous cell carcinoma (OSCC) is relatively uncommon but especially aggressive cancer. It is associated with a high rate of local recurrence and poor survival ${ }^{(3)}$. Like other cancers, OSCC denotes piling up of flaw in the genes that encode key proteins associated with growth and development. This manifests during a concatenation of precancerous stages, manifested morphologically as epithelial dysplasia or intraepithelial lesions in the sequence of dysplasia-carcinoma ${ }^{(4)}$. The increased prolifera-

* Assistant Professor of Oral Pathology - Faculty of Dentistry - Al-Azhar University.

** Lecturer of Oral Pathology - Faculty of Dentistry - Al-Azhar University.

*** Lecturer of Oral Histology - Faculty of Dentistry - Al-Azhar University. 
tive activity observed in OSCC compared to nonneoplastic hyperplasia. The mode of unprecedented remediation strategies, the development of early detection methods and the prediction of patient outcome depended upon identification of the specific gene proteins and the sequence in which they appear in the normal, premalignant and malignant cells ${ }^{(6)}$, p53 a tumor suppressor gene, is reported to be the most frequent target for genetic alterations leading to cancer. This gene regulates cell proliferation and DNA repair by inhibiting the cell cycle at G1/S phase; loss of p53 function may therefore lead to aberrant cell kinetics. Mutation of the p53 gene is thought to be a fundamental ingredient of oral carcinogenesis ${ }^{(7)}$.

Based on gene sequence homologies, a p53gene family has been identified that includes p53, p63 ${ }^{(8)}$, and p73 genes ${ }^{(9)}$, p63 protein has been detected in many human normal tissues including skin, esophageal mucosa, oral mucosa, lung, bladder, kidney, salivary glands, breast, exocervix, prostate, and tonsil ${ }^{(10-13)}$. It is known to play an essential role in epithelial development and the proliferation of limb and craniofacial structures ${ }^{(14,15)}$. p63 is expressed in multiplying cells, and is thought to be a marker for keratinocyte stem cells ${ }^{(16)}$. During the G2- and Mphases of actively dividing cells a nuclear protein ki67 is expressed. This antigen is a proliferation marker that correlates with the existence and seriousness of epithelial dysplasia. It provides considerable datum on the degree of aggressiveness and ultimatum of $\operatorname{OSCC}^{(17)}$.

In oral mucosal lesions, the k67 is considered as a good marker of cellular proliferation in premalignant and malignant lesions and is informed as a marker of the presence and severity of oral intraepithelial lesions, this is a result of the expression of ki67 which reported to increase according to the proliferative activity and degree of epithelial dysplasia ${ }^{(18)}$. The aim of this study was to investigate the expression of $\mathrm{p} 63$ protein and ki67 antigen in a series of graded OSCC to evaluate the potential association between the expression of the two markers and the aggressiveness of histologic grades of OSCC.

\section{MATERIALS AND METHODS:}

Thirty of paraffin blocks consisting of different types of OSCC (10 cases of well differentiated OSSC, 10 cases of moderately differentiated OSSC, 10 cases of poorly differentiated OSSC) will be collected from Department of Oral and Maxillofacial Surgery, Faculty of Dental Medicine, Al-Azhar University. The all sections will be stained with hematoxylin and eosin (H\&E) and immunohistochemical (IHC) marker for detection of p63 \& ki67 proteins. The immunostaining procedure was performed according to the manufacturer instructions, $3 \mu \mathrm{m}$ thick sections were made on poly-l-lysine $(0.1 \%$ [w/v] in $\mathrm{H} 2 \mathrm{O})$ (Sigma-Aldrich, Missouri, USA) coated slides. Sections were deparaffinized and rehydrated with xylene and serial dilutions of ethanol to distilled water. Tissue sections were immersed in EDTA buffer at a $\mathrm{pH}$ of 9 and heat-induced epitome retrieval was done using autoclave method at $120^{\circ} \mathrm{C}, 12-15 \mathrm{psi}$ for $15 \mathrm{~min}$. Primary antibodies against the respective proteins were added (ki67 1:100, p63 1:200 Dako, Carpinteria, CA, USA) and incubated overnight at $4^{\circ} \mathrm{C}$ in a humidified chamber. After rinsing with phosphate buffered saline (PBS), slides were incubated with secondary antibody followed by streptavidin-biotin-peroxidase complex, both for 30 min at room temperature with a PBS wash between each step; all tissue slides were counterstained using haematoxylin stain. The immunostained sections were studied using light microscope to evaluate the propagation of positive cases and the localization of immmunostaining within the tissues. A section was considered either positive or negative according to the presence or absence of brown staining in the nuclei of tumor cells regarding p63, and in cytoplasm of tumor cells regarding ki 67 analysis. 
In addition, image analysis computer system was used to assess area percentage of positive cells of the immunostaining. This was done in the Oral and Dental Pathology Department - Faculty of Dental Medicine - Boys- Cairo - Al-Azhar University. The degree of positive staining for each antibody was evaluated by a well-established semi-quantitative scoring on a scale range from negative to strong positive staining as follow: Strong staining (more than $50 \%$ stained), moderate staining (between 25 and $50 \%$ stained), weak staining (between 5 and $25 \%$ stained), and negative (less than $5 \%$ stained) ${ }^{(19)}$. Statistical presentation and analysis of the present study was conducted, using the mean, standard deviation, ANOVA, Tukey's test and Spearman's correlation coefficient was used to determine correlations between different measurements by SPSS V204. Significant level: Non significant $>0.05$, significant $<0.05^{*}$ high significant $<0.001^{*}$.

\section{RESULT:}

\section{Histopathological and immunohistochemical results}

$\mathrm{H}$ \& $\mathrm{E}$ stain for the 10 cases of well differentiated OSSC tissue sections showed nests of neoplastic epithelial cells in connective tissue, cells of the invading nests having pleomorphism, hyperchromatism and abnormalmitosis and one of the prominent features is presence numerous epithelial \&/ or keratin pearls (Fig.1, A) IHC staining using p63 of well differentiated OSSC tissue sections showed weak $(18.13 \%)$ positive nuclear expression throughout the peripheral cells of the invading nests (Fig.1, B), while ki67 expression exhibited weak $(23.08 \%)$ positive cytoplasmic expression throughout the peripheral cells of the invading nests (Fig.1, C). H \& E stain for the 10 cases of moderately differentiated OSSC tissue sections showed nests of neoplastic epithelial cells in connective tissue, cells of the invading nests having pleomorphism, hyperchromatism and abnormalmitosis and, few keratin \& epithelial pearls were observed (Fig.2, A) IHC staining using p63 moderately differentiated OSSC tissue sections showed moderate $(44.73 \%)$ positive nuclear expression throughout the peripheral and central cells of the invading nests (Fig.2, B), while ki67 expression exhibited strong (57.07\%) positive cytoplasmic expression throughout the peripheral and central cells of the invading nests (Fig.2, C). H \& E stain for the 10 cases of poorly differentiated OSSC tissue sections showed nests of neoplastic epithelial cells in connective tissue, cells of the invading nests having pleomorphism, hyperchromatism and abnormalmitosis and the cells are scattered individually \& no keratin or epithelial pearls were observed (Fig.3, A) IHC staining using p63 poorly differentiated OSSC tissue sections showed strong $(60.31 \%)$ positive nuclear expression throughout scattered neoplastic cells (Fig.3, B), while ki67 expression exhibited strong (71.92 $\%)$ positive cytoplasmic expression throughout scattered neoplastic cells (Fig.3, C).

\section{Statistical analysis results}

Statistical analysis results of p63 \& ki67 expression were obtained by comparing the area $\%$ between different grading of OSSC, statistical analysis results were revealed that, in regard to expression of p63 \& ki67, poorly differentiated OSSC had recorded the highest mean area $(60.31 \%)$, $(71.92 \%)$, respectively while well differentiated OSSC had recorded the lowest mean area (18.13 $\%),(23.08 \%)$, respectively and the comparison revealed that there was high significant difference between well \& moderately differentiated OSSC also between well $\&$ poorly differentiated OSSC where $\mathrm{P}$ value was $<0.001$. There was statistically significant difference between moderately \& poorly differentiated OSSC where $\mathrm{P}$ value was $<0.05$ (Table 1and Fig. 4). There was a statistically significant direct correlation between p63 \& ki67 stain i.e. an increase in p63is associated with an increase in ki67stain and vice versa (Table 1and Fig. 5). 


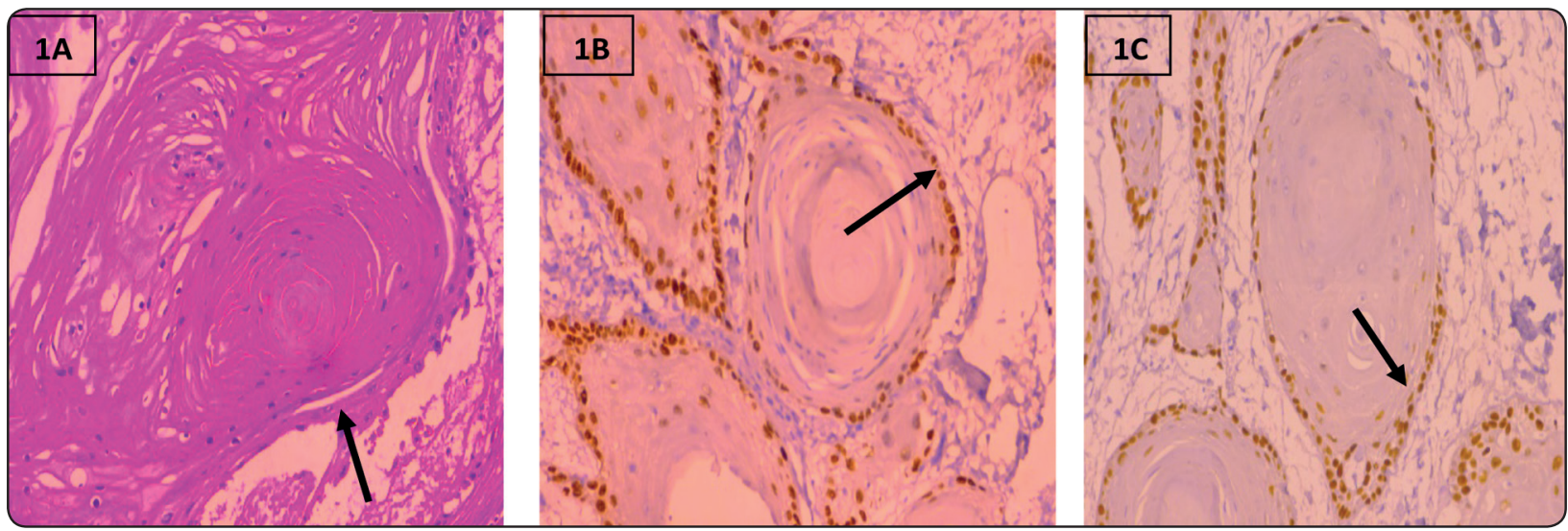

Fig. (1) A: H\&E stain of well differentiated OSSC sections showed neoplastic epithelial nests with keratin pearls invading the C.T (arrow) (x 200). Fig.1, B: p63 showed positive nuclear expression in the infiltrative margin of well differentiated OSSC was found in the peripheral area of tumor nest (arrow) (x200). Fig.1, C: ki-67 showed positive cytoplasmic expression in the infiltrative margin of well differentiated OSSC was found in the peripheral area of tumor nest (arrow) (x200).

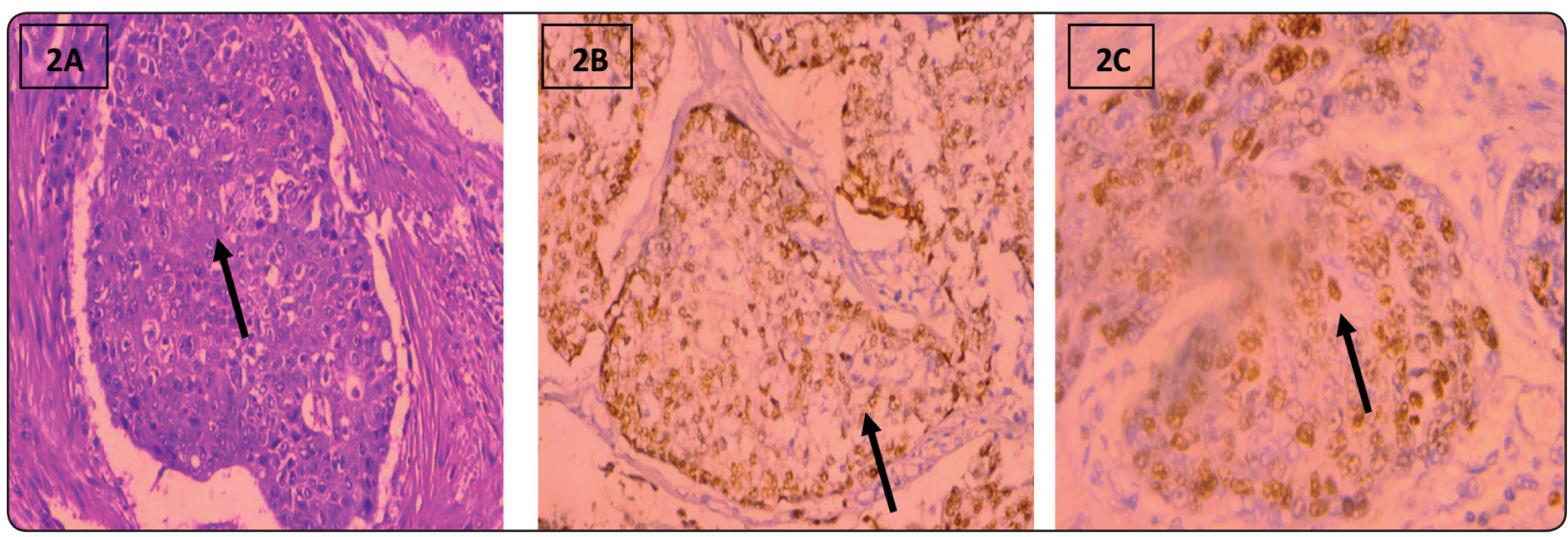

Fig. (2) A: H\&E stain of moderately differentiated OSSC sections showed neoplastic epithelial nests with keratin pearls invading the C.T. (arrow) (x 200). Fig.2, B: p63 showed positive nuclear expression of moderately differentiated OSSC was found in the peripheral and central cells of tumor nest (arrow) (x200). Fig.2, C: ki-67 showed positive cytoplasmic expression of moderately differentiated OSSC was found in the peripheral and central cells of tumor nest (arrow) (x400).

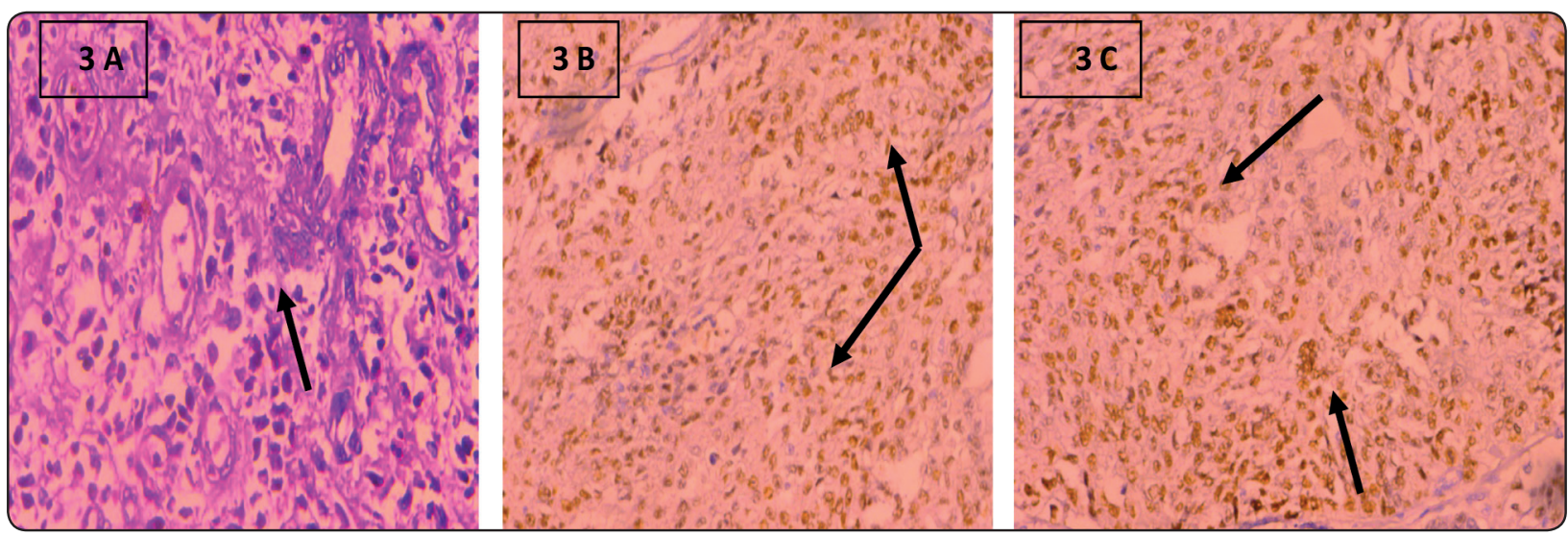

Fig. (3) A: H\&E stain of poorly differentiated OSSC the neoplastic cells are scattered individually \& no keratin or epithelial pearls were observed (arrow) (x 100). Fig.3, B: p63 showed positive cytoplasmic expression of poorly differentiated OSSC throughout scattered neoplastic cells (arrows) (x100). Fig.3, C: ki-67 showed positive cytoplasmic expression of poorly differentiated OSSC throughout scattered neoplastic cells (arrows) (x100). 
TABLE (1): Mean standard deviation (SD), correlation coefficient (r) and P- values of p63 and Ki-67 stains in different types of OSSC.

\begin{tabular}{|l|l|l|l|l|l|}
\hline \multirow{2}{*}{ OSSC } & \multicolumn{2}{l|}{ p63 } & Ki-67 \\
\cline { 2 - 6 } & Mean & SD & Mean & SD \\
\hline \multirow{2}{*}{ Well differentiated type } & 18.13 & 3.84 & 23.08 & 7.58 \\
\hline Moderately differentiated type & 44.73 & 12.21 & 57.07 & 12.71 \\
\hline \multirow{2}{*}{ Poorly differentiated type } & 60.31 & 8.90 & 71.92 & 17.43 \\
\hline \multirow{3}{*}{ ANOVA } & $\mathrm{f}$ & 56.161 & 35.979 \\
\hline & P-value & $<0001^{* *}$ & $<0001^{* *}$ \\
\hline \multirow{3}{*}{ Tukey's test } & Well \& Moderately differentiated OSSC & $<0001^{* *}$ & $<0001^{* *}$ \\
\cline { 2 - 5 } & Well \& Poorly differentiated OSSC & $<0001^{* *}$ & $<0001^{* *}$ \\
\cline { 2 - 6 } & Moderately \& Poorly differentiated OSSC & $0.002^{*}$ & $0.046^{*}$ & \\
\hline
\end{tabular}

$\underline{\text { Ki-67 }}$

$\underline{\mathrm{p63}}$

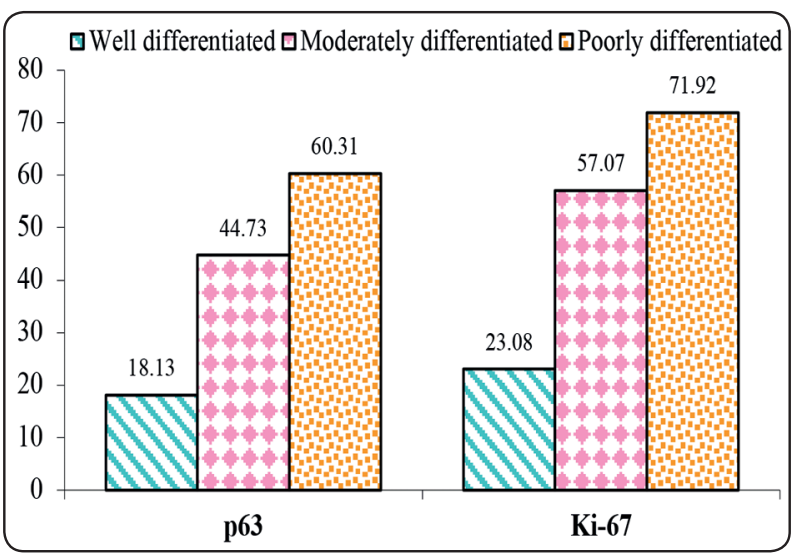

Fig. (4) Bar chart representing mean of p63 and Ki-67 stains in different types of OSSC.

\section{DISCUSSION}

Tumor initiation, progression and invasion involve cellular proliferation, apoptosis as well as cell adhesion and communication that ensure cell survival, renewal and co-ordination ${ }^{(20)}$. Like other cancers, OSCC represents a piling up of disorder in the genes that encode key proteins associated

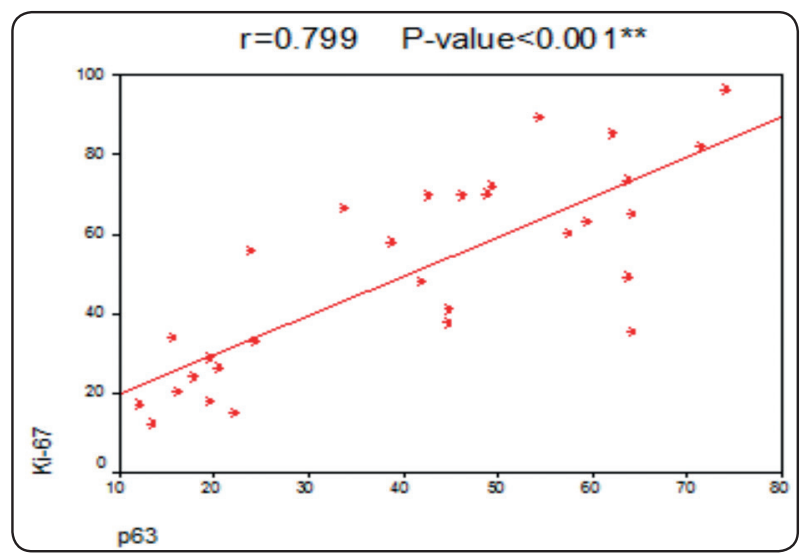

Fig. (5) Scatter diagram representing direct correlation between p63 and Ki-67 stains.

with growth and development. This appears through a series of precancerous stages, manifested morphologically as epithelial dysplasia or intraepithelial lesions in the sequence of dysplasiacarcinoma ${ }^{(21)}$. In the present study, we evaluated ki67 and p63 overexpression in biopsy specimens of all OSCC. Ki67 is one of the mitotic indicators in proliferative activity of tumors, expression of ki67 
in mean of proliferative activity of tumor cells is one of the indicators for tumor invasion potential and invasive activity of cancers related to degree of malignant neoplastic cells ${ }^{(22)}$. In the present study there were high significant difference among the mean of area percentage of ki67 between different grade of OSCC ( $p \leq 0.001)$, this results in agreement with previous studies, Sharmistha et al (2014) (23) observed $83.33 \%$ of OSCC cases were positive for ki67, Raju et al (2005) ${ }^{(24)}$ observed ki67 expression in $93.10 \%$ of premalignant lesion and $90 \%$ of oral cancer. Motta et al (2009) showed $92.75 \%$ cases with ki-67 expression ${ }^{(22)}$. Takkem et al (2005) ${ }^{(25)}$ observed that cell proliferation in OSCC increasing according to histological grades by an antibody for ki-67 protein. Because of expression ki-67 protein in all proliferating cells and the prognostic value of the ki-67 marker in many cancers, ki-67 protein is a potential therapeutic target in cancer, and strategies that inactivate ki-67 protein are a promising antiproliferative approach, with potential applicability in cancer treatment ${ }^{(26)}$. In the present study. There were high significant difference among the mean of area percentage of p63 between different grade of OSCC ( $\mathrm{p} \leq 0.001)$, similar results were reported by Venkatesh et al (2018) ${ }^{(27)}$ who concluded that intended to evaluate whether the amount of p63 expression (expressed as percentage expression) could be related to any of the histological grading which is generally used to define the aggressiveness of the tumor such as the Broders' classification and Anneroth's multifactorial grading system, which takes into consideration various morphological and histological parameters previously mentioned. Loljung et al (2014) (28) found a significant correlation of p63 expression and patient survival in OSCCs, also Venkatesh et al (2018 ${ }^{(27)}$ reported that p63 to correlate with survival in OSCCs, where high expression was seen in tumors with poorer survival after treatment. Furthermore, the usage and importance of Anneroth's multifactorial grading system over Broders' grading system in routine histopathological reporting for incisional biopsies of OSCCs is stressed. p63 expression has been suggested to have an oncogenic role in the cell proliferation changes observed during carcinogenesis ${ }^{(29)}$. In current study, there significant direct correlation between p63 \& ki67 stain, an increase in p63 is associated with an increase in mean area percentage of ki67and vice versa $=0.799$ ) $(\mathrm{P} \leq 0.001)$, co-expression and association between p63 and ki-67 in OSCC were demonstrated in our study, suggesting that alterations in the p63 protein might lead to increased cell proliferation. In agreement with our findings Vered et al (2009) ${ }^{(30)}$ found a correlation between Ki-67 and P63 immunoreaction in epithelial dysplasia and OSCCs, also Sunit et al (2017) ${ }^{(31)}$ concluded that expression of p63 correlates with poor histological grades of OSCC and leukoplakia. The increased p63 expression parallel with the severity of lesions likely reflects the intense proliferative activity and invasiveness of these lesions. In general, cyclin D1and p63 expression increased with increased grade of dysplasia in leukoplakia and OSCC.

\section{CONCLUSION}

In our study we concluded that p63 \& ki67 could reflect biological features, such as proliferation and differentiation, for recognition of clinical behavior of OSCC also p63 \& ki67 alteration may be associated with the progression of these neoplasms.

\section{REFERENCES}

1. Barnes L, Eveson JW, Reichart P, Sidransky D. Editors. World Health Organization Classification of Tumours. Patholgy \& Genetics of Head and Neck Tumours. Lyon: IARC Press; 2005. 430 p.

2. Mithani SK, Mydlarz WK, Grumbine FL, Smith IM, Califano JA. Molecular genetics of premalignant oral lesions. Oral Dis. 2007;13: 126-33.

3. Brandizzi D, Gandolfo M, Velazco ML, Cabrini RL, Lanfranchi HE. Clinical features and evolution of oral cancer: A study of 274 cases in Buenos Aires, Argentina. Med Oral Patol Oral Cir Bucal. 2008; 13:544-48. 
4. Kumar V, Assas AK, Fausto N. Robbins P, Cotran W. Pathologic basis of disease. 8th ed. Philadelphia, PA: Elsevier Saunders. 2010.

5. Klieb HB, Raphael SJ. Comparative study of the expression of p53, Ki67, E-cadherin and MMP-1 in verrucous hyperplasia and verrucous carcinoma of the oral cavity. Head Neck Pathol. 2007; 1:118-22.

6. Montebugnoli L, Felicetti L, Gissi DB, Cervellati F, Servidio D, Marchetti C. Predictive role of p53 protein as a single marker or associated to Ki67 antigen in oral carcinogenesis. Open Dent J. 2008; 2:24-29.

7. Edström S, Cvetkovska E, Westin T, Young C. Overexpression of p53-related proteins predicts rapid growth rate of head and neck cancer. Laryngoscope. 2001; 111:124-30.

8. Yang A, Kaghad M, Wang Y, Gillett E, Fleming MD, Do“tsch V, et al. p63, a p53 homolog at 3q27-29, encodes multiple products with transactivating, death inducing and dominant-negative activities. Mol Cell. 1998; 2:305-16.

9. Kaghad M, Bonnet H, Yang A, Creancier L, Biscan J-C, ValentA, et al. Monoallelically expressed gene related to p53 at 1p36, a region frequently deleted in neuroblastoma and other humancancers. Cell. 1997; 90:809-19.

10. Bilal H, Handra-Luca A, Bertrand J-C, Fouret PJ. p63 is expressed in basal and myoephitelial cells of human normal and tumor salivary gland tissues. J Histoc Cytoch. 2003;51:133-39.

11. Di Como CJ, Urist MJ, Babayan I, Drobnjak M, Hedvat $\mathrm{CV}$, Teruya-Feldstein $\mathrm{J}$, et al. p63 expression profiles in human normal and tumor tissues. Clin Cancer Res. 2002; 8:494-501.

12. Hu H, Xia S-H, Li A-D, Xu X, Cai Y, Han Y-L, et al. Elevated expression of $\mathrm{p} 63$ protein in human esophageal squamous cell carcinomas. Int J Cancer. 2002;102:580-83.

13. Tsujita-Kyutoku M, Kiuchi K, Danbara N, Yuri T, Senzaki H, Tsubura A. p63 expression in normal human epidermis and epidermal appendages and their tumors. J Cut Pathol. 2003; 30: 11-17.

14. Mills AA, Zheng B, Wang X-J, Vogel H, Roop DR, Bradley A. p63 is a p53 homologue required for limb and epidermal morphogenesis. Nature. 1999; 398:708-13.

15. Yang A, Schweitzer R, Sun D, Kaghad M, Walker N, Bronson RT, et al. P63 is essential for regenerative proliferation in limb, craniofacial and epithelial development. Nature. 1999;398: 714-18.
16. Pellegrini G, Dellambra E, Golisano O, Martinelli E, Fantozzi I, Bondanza S, et al. p63 identifies keratinocyte stem cells. PNAS. 2001; 98:3156-61.

17. Tumuluri V, Thomas GA, Fraser IS. Analysis of the Ki67 antigen at the invasive tumour front of human oral squamous cell carcinoma. J Oral Pathol Med. 2002; 31:598-604.

18. Bjarnason GA, Jordan CK, Wood PA, Li Q, Lincoln DW, Sothern RB, et al.Circadian Expression of Clock Genes in Human Oral Mucosa and Skin, Association with Specific Cell-Cycle Phases. Am J Pathol. 2001; 158:1793-801.

19. Negi A, Puri A, Gupta R, Nangia R, Sachdeva A, Mittal M. Comparison of immunohistochemical expression of antiapoptotic protein survivin in normal oral mucosa, oral leukoplakia, and oral squamous cell carcinoma. Patholog Res Int 2015;2015:1-6.

20. Wicki A, Christofori G. The potential role of podoplanin in tumor invasion. Brit J Cancer. 2007; 96: 1-5.

21. Kumar V, Assas AK, Fausto N. Robbins P Cotran O. Pathologic Basis of disease. 8th ed. Philadelphia, PA: Elsevier Saunders. 2010.

22. Motta Rda R, Zettler CG, Cambruzzi E, Jotz GP, Berni RB. Ki-67 and p53 correlation prognostic value in squamous cell carcinomas of the oral cavity and tongue. Braz J Otorhinolaryngol. 2009; 75:544-49.

23. Sharmistha M, Kinjal A, Prashant R, Bhavna G, Rasik N, Swati G. expression of p53 and ki-67 in oral dysplasia and squamous cell carcinoma: an immunohistochemical study. International Journal of Medical Science and Public Health. 2014; 3: 1201-14.

24. Raju B, Mehrotra R, Oijordsbakken G, Al-Sharabi AK, Vasstrand EN, Ibrahim SO. Expression of p53, Cyclin D1and Ki-67 in Pre-malignant and Malignant Oral Lesions: Association with Clinicopathological Parameters. Anticancer Res. 2005; 25:4699- 706.

25. Takkem A1, Barakat C, Zakaraia S, Zaid K, Najmeh J, Ayoub M, et al. Ki-67 Prognostic Value in Different Histological Grades of Oral Epithelial Dysplasia and Oral Squamous Cell Carcinoma. Asian Pac J Cancer Prev. 2018;19:3279-86.

26. Kausch I, Lingnau A, Endl E, Sellmann K, Deinert I, Ratliff TL, et al. Antisense treatment against Ki-67 mRNA inhibits proliferation and tumor growth in vitro and in vivo. Int J Cancer. 2003; 105: 710-16. 
27. Venkatesh1 A, Wadhwan1 V, Aggarwal P, Reddy V, Sharma1 P, Gotur S, et al. Elevated p63 expression as an indicator for poorer prognosis in squamous cell carcinomas of the oral cavity: An immunohistochemical study. Indian Journal of Medical and Paediatric Oncology. 2018; 39: 146-52.

28. Loljung L, Coates PJ, Nekulova M, Laurell G, Wahlgren M, Wilms T, et al. High expression of p63 is correlated to poor prognosis in squamous cell carcinoma of the tongue. J Oral Pathol Med. 2014;43:14-19.

29. Jung SM, Lin HC, Chu PH, Wu HH, Shiu TF, Huang $\mathrm{SL}$, et al. Expression of cell cycle-regulatory proteins,
MIB-1, p16, p53, and p63, in squamous cell carcinoma of conjunctiva:not associated with human papillomavirus infection. Virchows Arch. 2006, 448:301-305.

30. Vered M, Allon I, Dayan D. Maspin, p53, p63, and Ki67 in epithelial lesions of the tongue: from hyperplasia through dysplasia to carcinoma. J Oral Pathol Med. 2009; 38: 314-320.

31. Sunit B P, Bhari S. Manjunatha, Vandana Shah, Nishit Soni, Rakesh Sutariya. Immunohistochemical evaluation of p63 and cyclin D1 in oral squamous cell carcinoma and leukoplakia. J Korean Assoc Oral Maxillofac Surg. 2017; 43: 324-30. 